\title{
Molecular behaviors in thin film lubrication-Part two: Direct observation of the molecular orientation near the solid surface
}

\author{
Ming GAO ${ }^{1}$, Haoyu LI ${ }^{1}$, Liran MA ${ }^{1, *}$, Yuan GAO ${ }^{1,2}$, Linwei MA ${ }^{3}$, Jianbin LUO ${ }^{1, *}$ \\ ${ }^{1}$ State Key Laboratory of Tribology, Tsinghua University, Beijing 100084, China \\ ${ }^{2}$ School of Metallurgy Engineering, Xi'an University of Architecture and Technology, Xi'an 710055, China \\ ${ }^{3}$ School of Materials Science and Engineering, Tsinghua University, Beijing 100084, China \\ Received: 26 July 2018 / Revised: 09 November 2018 / Accepted: 13 January 2019 \\ (C) The author(s) 2019.
}

\begin{abstract}
Over the past twenty years, thin film lubrication (TFL) theory has been used to characterize the molecular behaviors in lubrication films thinner than $100 \mathrm{~nm}$, effectively bridging the gap between elastohydrodynamic lubrication and boundary lubrication. Unfortunately, to date, the TFL molecular model proposed in 1996 has not been directly proven by experimental detection. Herein, a method based on surface-enhanced Raman spectroscopy was developed to show both the packing and orienting of liquid molecules in the TFL regime. By trapping liquid crystal molecules between a structured silver surface and a glass surface, molecular ordering states dominated by shear effect and surface effect were successfully distinguished. A nanosandwich structure consisting of an adsorbed layer, an ordered-molecule layer, and a fluid layer was demonstrated. Molecule imaging in TFL was achieved. Our results illustrate the molecular behaviors and lubrication mechanism in nanoconfined films and facilitate the lubrication design of nanoelectromechanical and microelectromechanical systems.
\end{abstract}

Keywords: thin film lubrication; molecular behaviors; nematic liquid crystal; surface-enhanced Raman spectroscopy; lubrication theory; nanosandwich structure

\section{Introduction}

Molecules confined in a nanogap present special properties different from those at the macroscale [1-4]. In the case of a confined liquid film, previous reports demonstrated that, when the thickness of a film steps down to the nanoscale, solid-like structured molecules are formed, the friction is reduced, and the viscosity is increased [5, 6]. In tribological systems, molecular behaviors have attracted much attention owing to their significant effect on lubrication performance [7-11]. Researchers have already proposed many models to understand the behaviors of these molecules.

In 1922, Hardy and Doubleday proposed that, exactly two layers of molecules exist between wetting sliders and they are highly oriented when others are squeezed out $[12,13]$. Subsequently, some models of multi-layer molecules were suggested, many of them resulted from friction or wear tests [14-16]. However, many researchers admitted that very thin films are difficult to study [17]. Georges et al. reported an "immobile layer" for hexadecane using surface force apparatus [18]. Owing to the ultrathin film interferometry, Spikes et al. directly observed a sub-nanometer liquid film and believed that there is no boundary film for hexadecane and both mono- and multi-layered structures exist for stearic acid within a nanogap without any layered orientations [19-22]. This updated technique suggested a layer structure by testing the thickness of the liquid film. These findings have resulted in a series of models built to approximate the realness of molecules within the gap; however, neither of the

* Corresponding authors: Liran MA, E-mail: maliran@mail.tsinghua.edu.cn; Jianbin LUO, E-mail: luojb@mail.tsinghua.edu.cn 
aforementioned models offers a reasonable explanation for our observations in the current work.

As a branch of nanotribology, thin film lubrication (TFL) offered an alternative to interpret some anomalies that cannot be predicted by classical lubrication theory [23-26]. In 1996, the TFL molecular model was developed by Luo et al. [23]. It proposed that different layers, composed of a fluidic film, ordered film, and adsorbed film, will be formed, and the lubricant molecules are differently oriented in each layer owing to the surface influence and shearing force. The set-up of TFL theory and related models outlined a comprehensive map for lubrication regimes while offering insight into the molecular pattern in a confined liquid film [27]. However, to date, it has been difficult to observe and further confirm the TFL model via traditional techniques, because differentiating interfacial molecular information from bulk liquid nanofilms in an actual fluidic/lubrication process is difficult [28]. Zhang et al., in Part One of this serial work, investigated the molecular behavior of additives in TFL in the contact region and the orientation of lubricant molecules in the fluid layer [29]. They observed that polar additive molecules exhibited an enrichment effect in the Hertz contact region when added to a nonpolar base oil. The enrichment of additive molecules enhanced the film-forming ability of the lubricant and led to a reduction in the friction coefficient up to $61 \%$. However, the orientation of lubricant molecules near the solid surface is still unclear.

In this study, we develop a novel approach by combining the conventional Raman spectroscopy and surface-enhanced Raman spectroscopy (SERS). We observed that a thin Ag nanorod film deposited on a lower plano-convex surface could not only serve as an efficient SERS substrate to enhance the Raman intensity of interfacial molecules in the nanoconfined film dramatically but also induce a remarkable effect on the alignment of the adsorbed molecules [30]. Furthermore, we successfully distinguished the molecular information from the adsorbed layer and fluidic layer of the TFL. Our results demonstrate the likely influence of the surface energy and shearing force on the lubrication film. This work extends the applicability of the TFL model and initiates research on lubrication at the molecular level.

\section{Experimental details}

We employed a convex-on-disk friction test platform equipped with a Raman spectrometer as our basic experimental apparatus, 6CB (p-n-hexyl-p'-cyanobiphenyl,
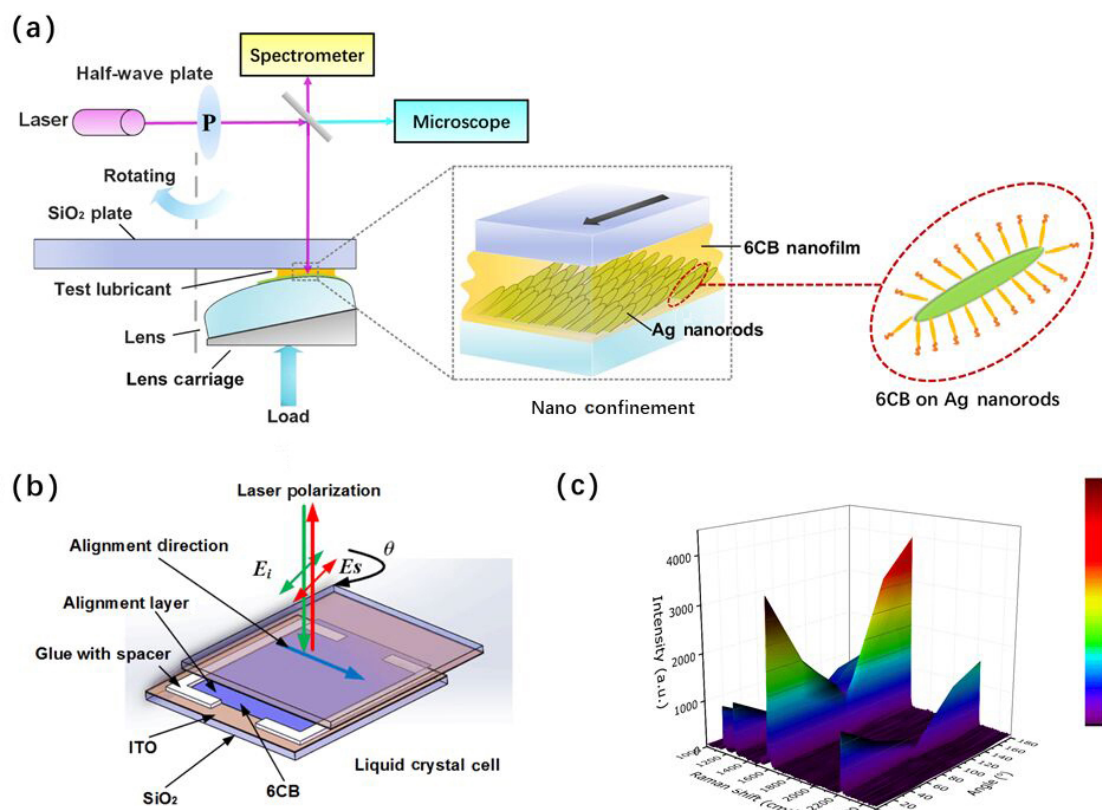

(c)

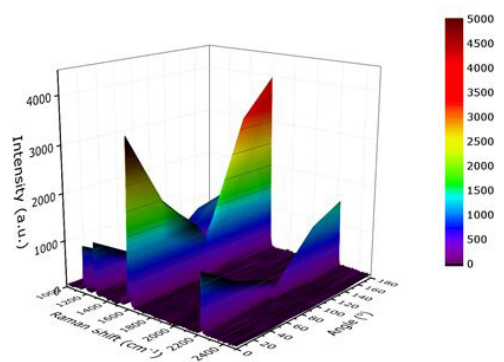

Fig. 1 (a) Schematic of the experimental apparatus and 6CB in the nanoconfinement, (b) illustration of an LC cell; $\theta$ is the angle between the direction of the polarized laser and the alignment direction of $6 \mathrm{CB}$, (c) Raman spectra of $6 \mathrm{CB}$ recorded with a laser polarized at different angles, where $\theta$ is marked for each line. 
$\mathrm{C}_{6} \mathrm{H}_{13}\left(\mathrm{C}_{6} \mathrm{H}_{6}\right)_{2} \mathrm{CN}$ ) was chosen as the testing lubricant. As a kind of rod-like uniaxial nematic liquid crystal (LC), 6CB represents a unique combination of ordering and fluidity at both macro- and nanoscale [31-35]. Owing to the essential nature of nematic LC, either planar or homeotropic structures could be tailored and detected easily [36-38]. Figure 1(a) shows the schematic of the experimental apparatus, where a rolling point contact condition was set up between the upper $\mathrm{SiO}_{2}$ plate (diameter: $100 \mathrm{~mm}$; thickness: $4 \mathrm{~mm}$ ) and the lower lens.

For the application of SERS, a Ag nanorod film was deposited through oblique angle deposition (OAD). A K9 plano-convex lens (purchased from Daheng Optics, China, $\phi=25.4 \mathrm{~mm}, R=90.44 \mathrm{~mm}, T=4 \mathrm{~mm}$ ), shown in extended Fig. 2(a), was used as the lower substrate. The fabrication of the Ag SERS substrate is shown in Fig. 2(b). To improve the binding capacity between the $\mathrm{Ag}$ nanorods and the K9 substrate, a 50-nm-thick flat Ti film was prepared on the substrate in advance through electron beam evaporation (GLAD, Thermionics, Inc.) [39, 40]. Subsequently, slanted Ag nanorods were deposited via the OAD technique in an electron-beam system. Here, the incident angle of the vapor flux $\alpha$ was set at $\sim 83^{\circ}$ off the surface normal of the substrates, with a deposition rate of $0.75 \mathrm{~nm} / \mathrm{s}$. The thickness of the Ag film is $500 \mathrm{~nm}$. A detailed procedure has been presented in previous reports [30]. Owing to the convex structure, the deposited Ag nanorods could only partially cover the substrate. As

(a)

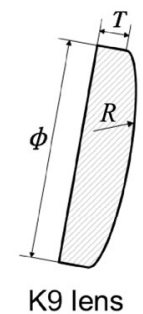

(c)

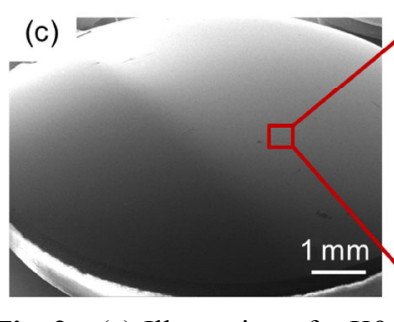

Fig. 2 (a) Illustration of a K9 plano-convex lens, (b) schematic of the OAD technique, (c) SEM image of the K9 plano-convex covered with $\mathrm{Ti}$ and $\mathrm{Ag}$ films, (d) SEM image of Ag nanorods. shown in Fig. 2(c), a boundary line between the Ti film (dark) and Ag film (bright) could be observed. According to the principles of the OAD technique, the growth direction of the Ag nanorods is perpendicular to the boundary line. A scanning electron microscopy (SEM) image of the Ag nanorods is displayed in Fig. 2(d).

To ensure that the contact area is located in the Ag-coated region, the plano-convex lens was supported by a wedge-shaped lens carriage. By applying a load on the lens against the $\mathrm{SiO}_{2}$ plate, a nanoscale $6 \mathrm{CB}$ absorption film was formed between the lens and $\mathrm{SiO}_{2}$ plate. The thickness of the absorption film was measured via a previously developed technique of relative optical interference intensity [41]. According to the Hamrock-Dowson formula, the thickness of the $6 \mathrm{CB}$ film in the contact center is proportional to the shearing rate [42].

An in situ Raman spectrometer equipped with an optical microscope was employed for studying the alignment behavior of $6 \mathrm{CB}$. The Raman signal of $6 \mathrm{CB}$ was collected in backscattering geometry $\left(180^{\circ}\right.$ scattering angle) using a He-Ne laser $(\lambda=514 \mathrm{~nm}$ emission wavelength) as the excitation source. The orientation of the $6 \mathrm{CB}$ molecules was measured using a polarized laser. Based on the results reported by Zhang et al., a single 6CB molecule could be regarded as having a uniaxial and rod-like structure [43]. As the major component of the $1,606 \mathrm{~cm}^{-1}$ mode in the Raman spectrum is parallel to the molecular axis, the intensity of $1,600 \mathrm{~cm}^{-1}$ changes with the angle of the polarized laser when the $6 \mathrm{CB}$ molecules are aligned in a specific direction. A LC cell was employed to detect the relationship between the intensity of $1,600 \mathrm{~cm}^{-1}$ and the direction of the polarized laser, as shown in Fig. 1(b). The angle between the polarized light and the direction of the alignment layer was defined as $\theta .6 \mathrm{CB}$ was added into the cell, and its molecules were oriented along the blue arrow marked in Fig. 1(b). The Raman spectra of 6CB recorded with a laser polarized at different angles are displayed in Fig. 1(c). They show that, when the polarization of the laser is perpendicular to the molecular axis $\left(\theta=90^{\circ}\right)$, the intensity of the peak at $1,606 \mathrm{~cm}^{-1}$ is at a minimum, whereas when the polarization of the laser is parallel to the molecular axis $\left(\theta=0^{\circ} / 180^{\circ}\right)$, the intensity is at a maximum. Thus, the orientation of $6 \mathrm{CB}$ could be 
obtained by calculating the polarization anisotropy of the $\mathrm{C}-\mathrm{C}$ aromatic vibration mode of $6 \mathrm{CB}$ at $1,606 \mathrm{~cm}^{-1}$. Moreover, the ratio of the maximum intensity and minimum intensity at a given wavenumber, which indicates the degree of order of the molecules, can be defined as $S$ (index of degree of order). Theoretically, the value of $S$ ranges from 1 to infinity. When $S$ is 1 , the molecules are completely disordered, without any specified orientation. The larger the value of $S$, the more ordered the molecules are. However, practically, limited by the noise baseline of the instrument, even for well-ordered molecules like the molecules in a LC cell, the value of $S$ is only 4.56 .

In a typical experimental process, the applied normal load is $0.2 \mathrm{~N}$, resulting in a nanocell diameter of $150 \mu \mathrm{m}$. Raman spectra were recorded with a spectral resolution of $2 \mathrm{~cm}^{-1}$ for values of the angle $\theta$ (formed by the direction of light polarization and the direction of rotating speed) varying from $0^{\circ}$ up to $360^{\circ}$ in steps of $15^{\circ}$. The integral time for each spectrum was $10 \mathrm{~s}$, and the laser power was $150 \mathrm{~mW}$. For contrast, the same lenses without any deposited film but covered with a 500-nm-thick flat Ag film were also employed as substrates. The integral time for each spectrum was $60 \mathrm{~s}$ for the transparent $\mathrm{K} 9$ lens and $10 \mathrm{~s}$ for the $\mathrm{Ag}$ substrate, and the laser power was $150 \mathrm{~mW}$. In addition, various shearing speeds, such as $50 \mathrm{~mm} / \mathrm{s}, 10 \mathrm{~mm} / \mathrm{s}$, and $200 \mathrm{~mm} / \mathrm{s}$ were used, leading to different influences on the alignment performance of $6 \mathrm{CB}$.

\section{Results and discussion}

\subsection{Orientation of molecules adsorbed on Ag nanorods during lubrication}

Our new approach induced both the Ag nanorod surface and the shear-flow field to direct the alignment of the 6CB molecules. As shown in Fig. 3(a-1), measurement was performed in the $X-Y$ plane, which is parallel to the surface of the tribopairs. The directions of shearing and the Ag nanorods are both depicted; the $Y$-axis was defined as $0^{\circ}$, whereas the projection of the deposited Ag nanorods in the $X-Y$ plane was along the $X$-axis direction. Figure 3(a-2) displays the microscopic image of a point contact confined between the $\mathrm{Ag}$ nanorod film and $\mathrm{SiO}_{2}$ plate in the initial stage, with a laser focused on the red dot to collect the signal.
Figures $3(b-1)$ to $3(b-3)$ show the relative Raman intensity of the adsorbed $6 \mathrm{CB}$ molecules at $1,606 \mathrm{~cm}^{-1}$ during the shearing process. The Raman results show that, in the static state, the maximum Raman intensity is obtained when the laser polarization is perpendicular to the growth direction of the Ag nanorods, whereas the minimum Raman intensity is obtained when the laser polarization is along the direction of the nanorods. It is observed that the orientation of $6 \mathrm{CB}$ on the $\mathrm{Ag}$ nanorods was along the $0^{\circ}$ direction when the value of $S$ was 1.42. In the subsequent step, a shear-flow field was applied along the $Y$-axis by rotating the $\mathrm{SiO}_{2}$ plate at a speed of $100 \mathrm{~mm} / \mathrm{s}$. After shearing for $30 \mathrm{~s}$, the Ag film on the lens surface was slightly worn. Figure 3(b-2) shows the relative Raman intensity at this moment, with the radar graph showing that the $6 \mathrm{CB}$ molecules were now randomly oriented, when $S$ is approximately 1 . After rotating the plate at $100 \mathrm{~mm} / \mathrm{s}$ for approximately $400 \mathrm{~s}$, the Ag film on it entered into a steady state. Interestingly, in this case, the orientation of the measured 6CB molecules on the Ag nanorod film was along the $90^{\circ}$ direction, when the value of $S$ was 2.23, as shown in Fig. 3(b-3), rather than in the flow direction. It is concluded that the ordering state of the $6 \mathrm{CB}$ molecules may be greatly affected by the morphology of the Ag nanorods.

To verify the assumption described above, the morphology of the $\mathrm{Ag}$ nanorods was observed at different shearing states. Figure 3(c-1) displays the SEM graph of the Ag nanorods in the contact area at the initial state (Fig. 3(b-1)), Fig. 3(c-2) shows the SEM graph of the Ag nanorods when shearing was conducted for $30 \mathrm{~s}$ (Fig. 3(b-2)), during which the Ag nanorods were pressed down and randomly arranged. Figure 3(c-3) shows the SEM image of the Ag nanorods after shearing for $400 \mathrm{~s}$, showing that the surface of the Ag nanorods was flat. The SEM images indicate that the morphologies of the measuring points in Figs. 3(b-1) to 3(b-3) are distinct, which indicates that the surface structure of the Ag film, rather than the applied flow field, was the primary driving force orienting the molecules in the measured 6CB nanofilm.

\subsection{Orientation of molecules on different substrates}

The crucial question that arises now is whether this is a phenomenon specific to the $\mathrm{Ag}$ nanorod surface. 
$(a-1)$

(a-2)

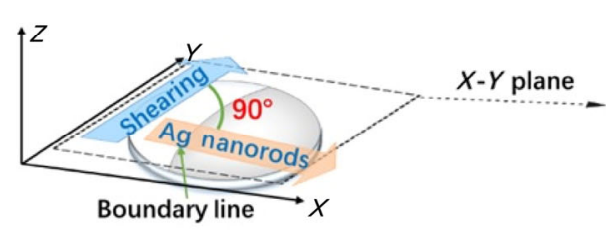

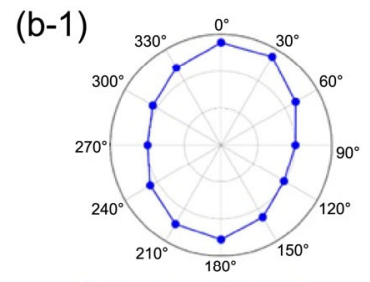

Static state (b-2)

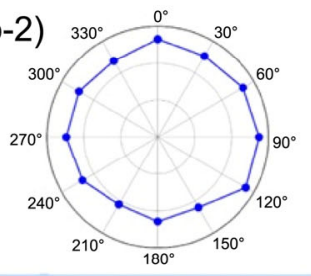

$30 \mathrm{~s}$ after shearing

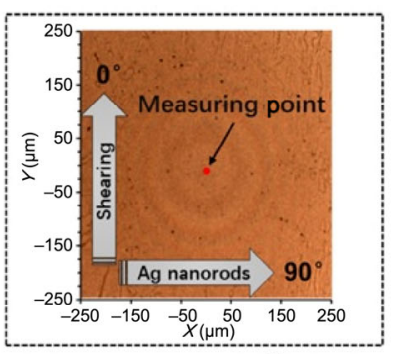

(b-3)

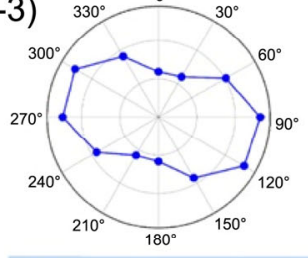

$400 \mathrm{~s}$ after shearing
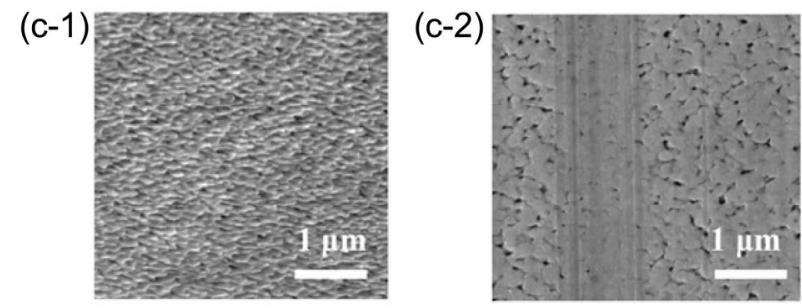

(c-3)

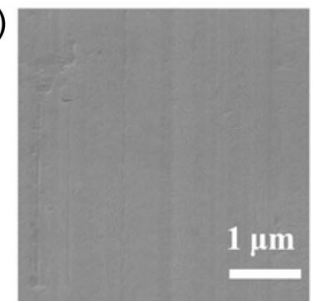

Fig. 3 (a-1) Schematics of a K9 plano-convex lens substrate covered with a Ag nanorod film under shearing, the $X$ - $Y$ plane was parallel to the surface, in-plane shearing was along the $Y$-axis, and the direction of the Ag nanorods was along the $X$-axis, here, the $Y$-axis was defined as $0^{\circ}$; (a-2) microscopic image of the contact area under shearing (rotating speed: $100 \mathrm{~mm} / \mathrm{s}$ ) at the initial state; (b) relative Raman intensity at different $\theta$ values (measuring point was marked as a red point in Fig. 3(a-2)), relative intensity of $1,600 \mathrm{~cm}^{-1}$ in the corresponding Raman spectra expressed via a radar graph at the initial state (Fig. 3(b-1)), after shearing for $30 \mathrm{~s} \mathrm{(Fig.} \mathrm{3(b-2))} \mathrm{and} \mathrm{after}$ shearing for $400 \mathrm{~s}$ (Fig. 3(b-3)); (c) SEM images of the Ag film around the measuring point before and after shearing: Fig. 3(c-1) initial static state, Fig. 3(c-2) after shearing for $30 \mathrm{~s}$, and Fig. 3(c-3) after shearing for $400 \mathrm{~s}$.

The behavior of $6 \mathrm{CB}$ on other substrates has been investigated during the shearing process, by applying a series of rotating speeds. A K9 plano-convex lens and a K9 plano-convex lens covered with a flat Ag film were used as bases for contrast, and the results are depicted in Fig. 4. Surprisingly, the shapes of the Raman radar graphs were different for the three groups. For the surface consisting of Ag nanorods aligned along the $X$-axis, the maximum relative Raman intensity was still aligned in the $90^{\circ}$ direction (Figs. 4(A-1)-4(A-3)); for the $\mathrm{K} 9$ lens surface, the maximum relative Raman intensity was aligned in the $0^{\circ}$ direction (Figs. 4(B-1)4(B-3)); whereas for the flat Ag surface, the relative Raman intensity was more or less equal at various polarized angles (Figs. 4(C-1)-4(C-3)).

It is generally accepted that a fluid film will be formed in the gap between two surfaces when a flow field is applied, and LC molecules prefer to align in the flow direction under shearing once they serve as the nanofilm [44]. However, notably, owing to the SERS effect, most of the Raman signal recorded at the $\mathrm{Ag}$ surface, for both the $\mathrm{Ag}$ nanorods and the flat $\mathrm{Ag}$ film, originates from the $6 \mathrm{CB}$ molecules adsorbed on or near the $\mathrm{Ag}$ surface $[45,46]$. This inference is drawn from the contrast between the intensities of the Raman spectra for the three substrates used, as shown in Fig. 5(a). The Raman spectra were collected under a shearing speed of $200 \mathrm{~mm} / \mathrm{s}$, and the integral time for each spectrum was $10 \mathrm{~s}$. We observed that, on the ordinary K9 lens, the Raman intensity was fairly low, but it could be enhanced up to 30 times or 103 times when a flat Ag film or Ag nanorod film were employed, respectively. This enhancement was caused by the SERS effect on the Ag nanostructure, and it has been reported that the enhanced range is approximately $5 \mathrm{~nm}$ near the surface [47]. Therefore, 


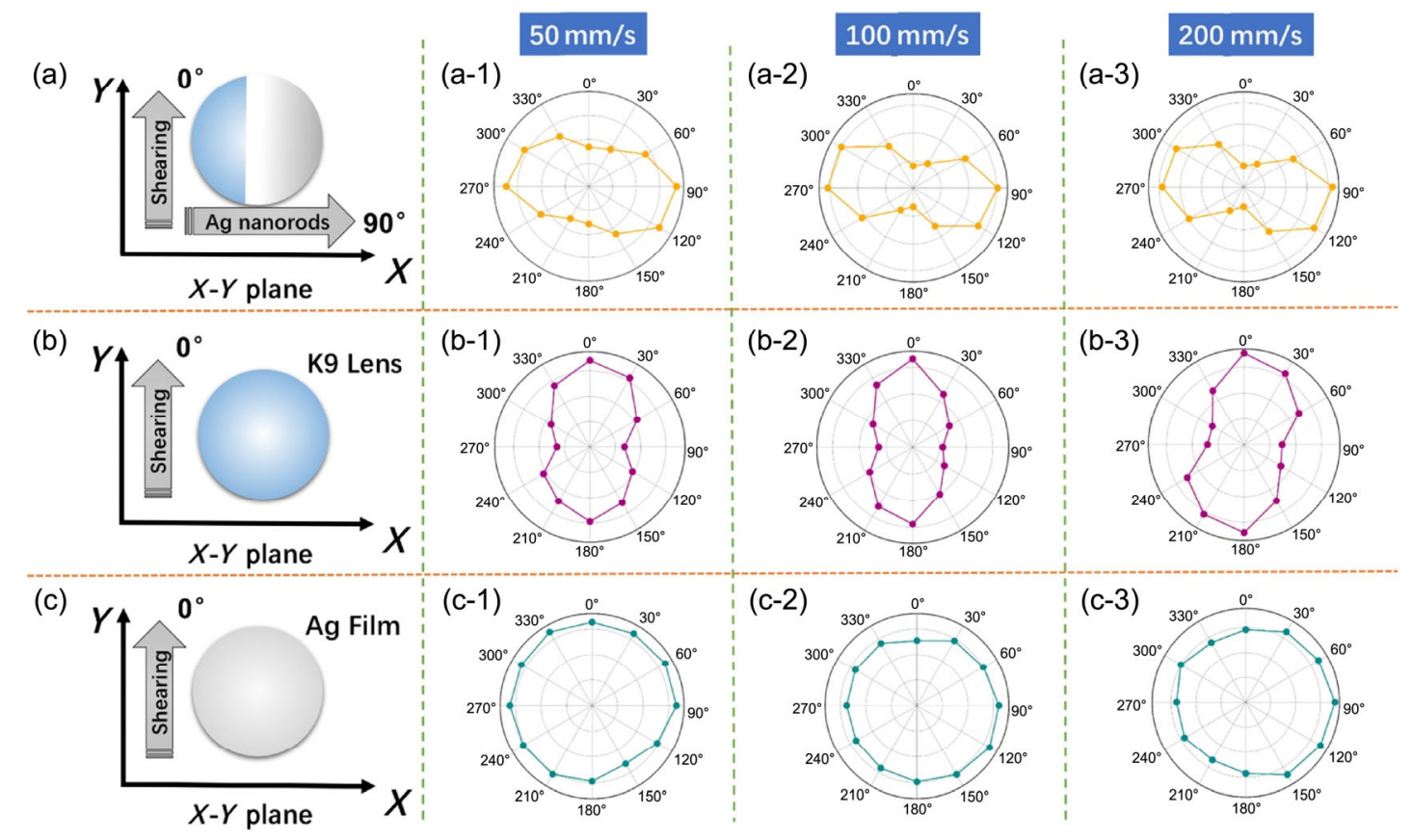

Fig. 4 (a-c) Illustration of the lower substrate. Relative Raman intensity graphs of 6CB on different substrates under shearing. The shear-flow speed is marked at the top of each pair. ((a-1)-(a-3)): performed on Ag nanorod film bases; ((b-1)-(b-3)): performed on K9 plano-convex lens; ((c-1)-(c-3)): performed on flat Ag film bases.

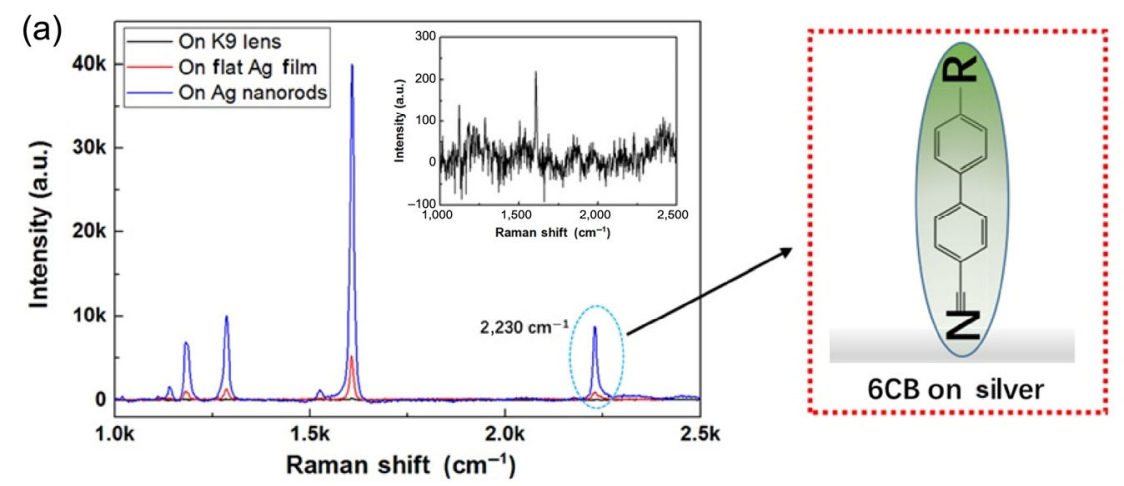

(b)

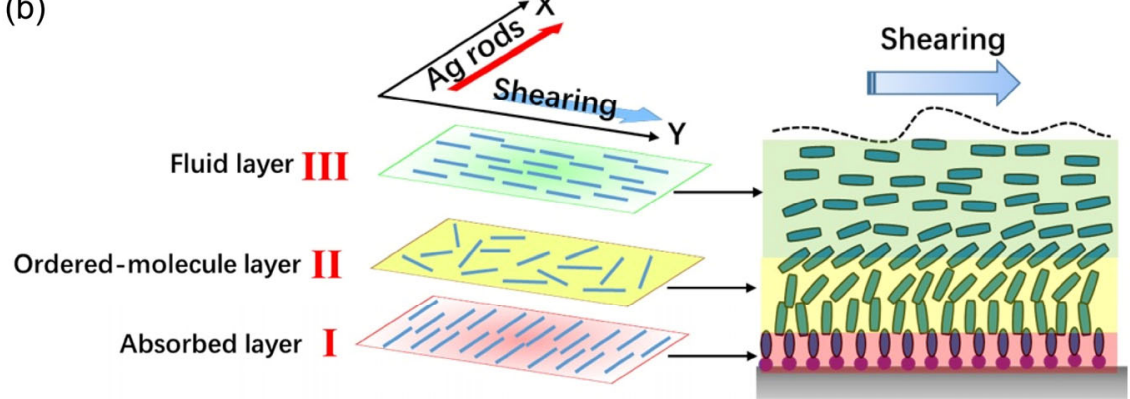

Fig. 5 (a) Raman spectra collected on the K9 lens, flat Ag film, and Ag nanorod film under shearing at a speed of $200 \mathrm{~mm} / \mathrm{s}$. The inset panel is the spectrum on the $\mathrm{K} 9$ lens. The adsorption of $6 \mathrm{CB}$ on $\mathrm{Ag}$ is depicted in the red rectangle; (b) illustration of $6 \mathrm{CB}$ alignment in a nanofilm under a shear-flow field.

even when a fluid film is formed, the information obtained through Raman spectra on the SERS substrate originates mostly from the adsorbed molecular layer on the surface. 


\subsection{Model of molecular orientation along the thickness of the lubricant film}

As indicated in Fig. 5(a), the peak at 2,230 $\mathrm{cm}^{-1}$ in the Raman spectra recorded on the SERS substrates was broadened, which shows that the cyano group (C-N) was influenced by $\mathrm{Ag}$ [48]. According to previous studies, 6CB molecules are inclined to be adsorbed vertically on the Ag surface via $\mathrm{C}-\mathrm{N}$ bonds as depicted in the inset panel in Fig. 5(a) [49]. Thus, in the initial static state, the alignment of the $6 \mathrm{CB}$ molecules was perpendicular to the projection of the Ag nanorods in the $X-Y$ plane, as per our measurements. During the shearing procedure, we assumed that the Ag nanorods had been pressed down and reoriented in the shearing direction, causing the $6 \mathrm{CB}$ molecules to be adsorbed onto the rubbed Ag nanorods aligned perpendicularly to the shearing direction, regardless of how the $\mathrm{Ag}$ nanorods were tilted initially.

Based on the above discussions, a layered molecular model demonstrating the molecular orientation in a nanofilm in the TFL regime was developed, as shown in Fig. 5(b). Near the surface, a thin adsorption film was formed, which is marked as the first layer I. In this case, the $6 \mathrm{CB}$ molecules were oriented perpendicularly to the Ag surface owing to the influence of surface adsorption, rather than the shearing force. The layer marked as III represents the fluid layer in the middle of the nanofilm. From the results collected on the $\mathrm{K} 9$ lens, it could be observed that $6 \mathrm{CB}$ in the fluid layer aligned along the flow direction. We supposed that, between layer I and layer III, an ordered-molecule layer (marked as layer II) would exist, and the molecules in this layer are oriented via an induction force. Thus, it could be summarized that the molecules under a flow field confined in a nanocell can orient in different ways; for molecules near or on surfaces, the ordering behavior is affected significantly by the surface adsorption, whereas the alignment of molecules in the middle fluidic layer is affected by the shearing force. This conclusion is consistent with the TFL model proposed by Luo et al. [23]. In this work, we have successfully demonstrated the layered molecular structure in TFL regime. Different materials will perform different lubrication characteristics. In the work reported in Ref. [50], a robust liquid superlubricity has been achieved in TFL regime, which facilitates the industrial applications of TFL theory.

\section{Conclusions}

In conclusion, we developed a method to control and to observe the alignment of nematic LC molecules confined in a nanogap. A lens-on-plate friction testing platform was used to create a nanocell with a $\mathrm{Ag}$ nanorod film deposited on the lower surface. The orientation of the $6 \mathrm{CB}$ molecules was determined by the growth direction of the $\mathrm{Ag}$ nanorods in the static state, caused by the vertical adsorption of the $6 \mathrm{CB}$ molecules on Ag through C-N bonds. Under a shearflow field, a layered molecular structure consisting of $6 \mathrm{CB}$ molecules was obtained. In the layered structure, the $6 \mathrm{CB}$ molecules aligned vertically near the surfaces but horizontally in the fluid layer. It was indicated that the Ag nanorods would be reoriented to the flow direction by the shearing force, resulting in the $6 \mathrm{CB}$ molecules adsorbed on it being aligned perpendicularly to the shearing direction. Finally, the molecules in the TFL regime could be successfully imaged. We believe that our findings will be crucial for studies on TFL and will facilitate the development of new methods for the in situ observation of molecular alignment patterns in films.

\section{Acknowledgements}

The work was financially supported by the National Natural Science Foundation of China (51305225, 51527901).

Ming GAO, Liran MA, and Jianbin LUO conceived and designed the study. Ming GAO performed the main experiments and Linwei MA fabricated the SERS substrate. Ming GAO, Haoyu LI, Liran MA, and Yuan GAO wrote the manuscript. Ming GAO, Liran MA, and Jianbin LUO reviewed and edited the manuscript. All the authors read and approved the manuscript.

Open Access This article is licensed under a Creative Commons Attribution 4.0 International License, which permits use, sharing, adaptation, distribution and reproduction in any medium or format, as long as you give appropriate credit to the original author(s) and the source, provide a link to the Creative Commons 
licence, and indicate if changes were made.

The images or other third party material in this article are included in the article's Creative Commons licence, unless indicated otherwise in a credit line to the material. If material is not included in the article's Creative Commons licence and your intended use is not permitted by statutory regulation or exceeds the permitted use, you will need to obtain permission directly from the copyright holder.

To view a copy of this licence, visit http://creativecommons.org/licenses/by/4.0/.

\section{References}

[1] Raviv U, Klein J. Fluidity of bound hydration layers. Science 297(5586): 1540-1543 (2002)

[2] Raviv U, Laurat P, Klein J. Fluidity of water confined to subnanometre films. Nature 413(6851): 51-54 (2001)

[3] Klein J, Kumacheva E. Confinement-induced phase transitions in simple liquids. Science 269(5225): 816-819 (1995)

[4] Gao J P, Luedtke W D, Landman U. Nano-elastohydrodynamics: Structure, dynamics, and flow in nonuniform lubricated junctions. Science 270(5236): 605-608 (1995)

[5] Granick S. Motions and relaxations of confined liquids. Science 253(5026): 1374-1379 (1991)

[6] Israelachvili J, Gourdon D. Putting liquids under molecular scale confinement. Science 292(5518): 867-868 (2001)

[7] Gao X L, Dai K, Wang Z, Wang T T, He J B. Establishing quantitative structure tribo-ability relationship model using Bayesian regularization neural network. Friction 4(2): 105-115 (2016)

[8] Berman D, Deshmukh S A, Sankaranarayanan S K, Erdemir A, Sumant A V. Macroscale superlubricity enabled by graphene nanoscroll formation. Science 348(6239): 11181122 (2015)

[9] Thompson P A, Robbins M O. Origin of stick-slip motion in boundary lubrication. Science 250(4982): 792-794 (1990)

[10] Ma L R, Zhang C H, Luo J B. Investigation of the film formation mechanism of oil-in-water $(\mathrm{O} / \mathrm{W})$ emulsions. Soft Matter 7(9): 4207-4213 (2011)

[11] Xie G X, Luo J B, Liu S H, Guo D, Zhang C H. "Freezing" of nanoconfined fluids under an electric field. Langmuir 26(3): 1445-1448 (2010)

[12] Hardy W B, Doubleday I. Boundary lubrication. - The paraffin series. Proc Roy Soc A Math Phys Eng Sci 100(707): 550-574 (1922)

[13] Hardy W B, Doubleday I. Boundary lubrication. - The latent period and mixtures of two lubricants. Proc Roy Soc A
Math Phys Eng Sci 104(724): 25-38 (1923)

[14] Stanton T E. Friction. London (UK): Longmans, Green and Company, 1923.

[15] Bowden F P, Tabor D. The friction and lubrication of solids. Oxford (UK): Oxford University Press, 2001.

[16] Bailey A I, Courtney-Pratt J. The area of real contact and the shear strength of monomolecular layers of a boundary lubricant. Proc Roy Soc A Math Phys Eng Sci 227(1171): 500-515 (1955)

[17] Spikes H A. Boundary lubrication and boundary Films. In Tribology Series. Dowson D, Taylor C M, Childs T H C, Godet M, Dalmaz G, Eds. Oxford: Elsevier, 1993: 331-346.

[18] Georges J M, Millot S, Loubet J L, Tonck A. Drainage of thin liquid films between relatively smooth surfaces. $J$ Chem Phys 98(9): 7345-7360 (1993)

[19] Johnston G J, Wayte R, Spikes H A. The measurement and study of very thin lubricant films in concentrated contacts. Tribol Tran 34(2): 187-194 (1991)

[20] Spikes H A. Direct observation of boundary layers. Langmuir 12(19): 4567-4573 (1996)

[21] Chinas-Castillo F. The behaviour of colloids in lubricated contacts. Master's thesis. London (UK): University of London, 2000.

[22] Ratoi M, Spikes H A, Bovington C. Langmuir-blodgett films in high-pressure rolling contacts. Tribol Trans 46(1): 24-30 (2003)

[23] Luo J B, Wen S Z, Huang P. Thin film lubrication. Part I. Study on the transition between EHL and thin film lubrication using a relative optical interference intensity technique. Wear 194(1-2): 107-115 (1996)

[24] Urbakh M, Klafter J, Gourdon D, Israelachvili J. The nonlinear nature of friction. Nature 430(6999): 525-528 (2004)

[25] Luo J B, Shen M W, Wen S Z. Tribological properties of nanoliquid film under an external electric field. $J$ Appl Phys 96(11): 6733-6738 (2004)

[26] Shen M W, Luo J B, Wen S Z, Yao J B. Nano-tribological properties and mechanisms of the liquid crystal as an additive. Chin Sci Bull 46(14): 1227-1232 (2001)

[27] Luo J B, Shen M W, Shi B, Wen S Z. Thin film lubrication and lubrication map. Chin J Mech Eng 36(7): 5-10 (2000)

[28] $\mathrm{Hu}$ Y Z, Granick S. Microscopic study of thin film lubrication and its contributions to macroscopic tribology. Tribol Lett 5: 81-88 (1998)

[29] Zhang S H, Qiao Y J, Liu Y H, Ma L R, Luo J B. Molecular behaviors in thin film lubrication- Part one: film formation for different polarities of molecules, Friction, accepted.

[30] Zhou Q, Liu Y J, He Y P, Zhang Z J, Zhao Y P. The effect of underlayer thin films on the surface-enhanced Raman 
scattering response of $\mathrm{Ag}$ nanorod substrates. Appl Phys Lett 97(12): 121902 (2010)

[31] Lagerwall J P F, Scalia G. A new era for liquid crystal research: applications of liquid crystals in soft matter nano-, bio-and microtechnology. Curr Appl Phys 12(6): 1387-1412 (2012)

[32] Woltman S J, Jay G D, Crawford G P. Liquid-crystal materials find a new order in biomedical applications. Nat Mater 6(12): 929-938 (2007)

[33] Ruths M, Steinberg S, Israelachvili J N. Effects of confinement and shear on the properties of thin films of thermotropic liquid crystal. Langmuir 12(26): 6637-6650 (1996)

[34] Cann P M, Aderin M, Johnston G J, Spikes H A. Paper V (iii) An investigation into the orientation of lubricant molecules in EHD contacts. Tribol Ser 21: 209-218 (1992)

[35] Nakano K. Scaling law on molecular orientation and effective viscosity of liquid-crystalline boundary films. Tribol Lett 14(1): 17-24 (2003)

[36] Gibbons W M, Shannon P J, Sun S T, Swetlin B J. Surface mediated alignment of nematic liquid crystals with polarized laser light. Nature 351(6321): 49-50 (1991)

[37] Körner H, Shiota A, Bunning T J, Ober C K. Orientation on-demand thin films: Curing of liquid crystalline networks in ac electric fields. Science 272(5259): 252-255 (1996)

[38] Kundu S, Lee M H, Lee S H, Kang S W. In situ homeotropic alignment of nematic liquid crystals based on photoiso merization of Azo-Dye, physical adsorption of aggregates, and consequent topographical modification. Adv Mater 25(24): 3365-3370 (2013)

[39] Ma L W, Huang Y, Hou M J, Li J H, Xie Z, Zhang Z J. Pinhole-containing, subnanometer-thick $\mathrm{Al} 2 \mathrm{O} 3$ shell-coated $\mathrm{Ag}$ nanorods as practical substrates for quantitative surface enhanced Raman scattering. J Phys Chem C 120(1): 606-615 (2016)
[40] Ma L W, Wu H, Huang Y, Zou S M, Li J H, Zhang Z J. High-performance real-time SERS detection with recyclable ag nanorods@HfO2 substrates. ACS Appl Mater Interfaces 8(40): 27162-27168 (2016)

[41] Ma L R, Zhang C H. Discussion on the technique of relative optical interference intensity for the measurement of lubricant film thickness. Tribol Lett 36: 239-245 (2009)

[42] Dowson D, Hopkins D W, Higginson G R. Elasto hydrodynamic lubrication: International series on materials science and technology. Amsterdam (UK): Elsevier, 2014.

[43] Zhang S H, Liu Y H, Luo J B. In situ observation of the molecular ordering in the lubricating point contact area. $J$ Appl Phys 116(1): 014302 (2014)

[44] Gähwiller C. Temperature dependence of flow alignment in nematic liquid crystals. Phys Rev Lett 28(24): 1554-1556 (1972)

[45] Stockman M I. Spasers explained. Nat Photon 2(6): 327-329 (2008)

[46] Barnes W L, Dereux A, Ebbesen T W. Surface plasmon subwavelength optics. Nature 424(6950): 824-830 (2003)

[47] Hao E C, Schatz G C. Electromagnetic fields around silver nanoparticles and dimers. $J$ Chem Phys 120(1): 357-366 (2004)

[48] Perrot M, De Zen J M, Rothschild W G. Mid-and low frequency Raman spectra of stable and metastable crystalline states of the 4-n-alkyl-4'-cyanobiphenyl $(n=9,11,12)$ liquid crystals. J Raman Spectrosc 23(11): 633-636 (1992)

[49] Gray G W, Mosley A. The raman spectra of 4-Cyano-4'pentylbiphenyl and 4-Cyano-4'-pentyl-d11-biphenyl. Mol Cryst Liq Crys 35(1-2): 71-81 (1976)

[50] Ge X Y, Halmans T, Li J J, Luo J B. Molecular behaviors in thin film lubrication-Part three: Superlubricity attained by polar and nonpolar molecules. Friction, DOI 10.1007/ s40544-018-0254-2.

China. Afterwards she pursued her Ph.D degree in the State Key Laboratory of Tribology at Tsinghua University, Beijing, China and got her Ph.D degree in mechanical engineering in 2016. Her research interest is molecular behaviors in thin film lubrication. 


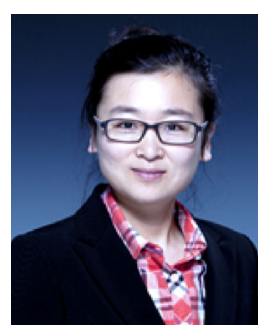

Liran MA. She received her BS degree from Tsinghua University in 2005, and received her Ph.D degree from Tsinghua University in 2010. Following a postdoctoral period at the Weizmann Institute of Science in Israel, she is now working as an associate professor in State Key Laboratory of Tribology, Tsinghua University. Her interests in tribology

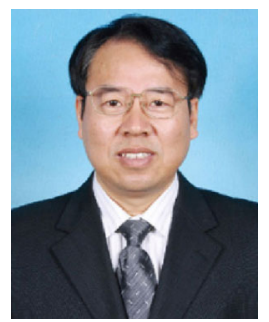

Jianbin LUO. He received his BS degree from Northeastern University in 1982, and got his MS degree from Xi'an University of Architecture and Technology in 1988. In 1994, he received his Ph.D degree from Tsinghua University and then joined the faculty of Tsinghua University. Prof. Jianbin LUO is an Academician of the Chinese Academy of Sciences and a Yangtze River Scholar Distinguished have ranged from aqueous lubrication and hydration lubrication to the liquid/solid interface properties. She has published over 50 papers. Her work has been cited some 300 times. Her honors include the Hinwin Doctoral Dissertation Award (2011), the Maple leaf award for Outstanding Young Tribologists (2015), and Chang Jiang Scholars Program-Young Professor Award (2015).

Professor of Tsinghua University, Beijing, China. He was awarded the STLE International Award (2013), the Chinese National Technology Progress Prize (2008), the Chinese National Natural Science Prize (2001), and the Chinese National Invention Prize (1996). Prof. LUO has been engaged in the research of thin film lubrication, superlubricity and tribology in nanomanufacturing. He was invited as a keynote or plenary speaker for more than 20 times on the international conferences. 